\title{
Partial hydatidiform mole progression into invasive mole with lung metastasis following in vitro fertilization
}

\author{
XI ZHOU ${ }^{1}$, YONGLI CHEN ${ }^{1}$, YONGMEI LI $^{1}$ and $\mathrm{ZHAO} \mathrm{DUAN}^{2}$ \\ ${ }^{1}$ Department of Obstetrics and Gynecology, Renmin Hospital, Hubei University of Medicine, Shiyan, Hubei 442000; \\ ${ }^{2}$ Department of Obstetrics and Gynecology, The Second Affiliated Hospital of Xi'an Jiaotong University, \\ Xi'an, Shaanxi 710004, P.R. China
}

Received September 6, 2011; Accepted November 23, 2011

DOI: $10.3892 / \mathrm{ol} .2011 .542$

\begin{abstract}
In the present study, the case of a 34-year-old Chinese female who underwent in vitro fertilization resulting in a twin pregnancy was reported. Following in vitro fertilization, the patient was found to have a partial hydatidiform mole (PHM) with a co-existing twin by transvaginal sonography (TVS). At 16 weeks, the pregnancy was terminated and a normallooking fetus with a HM placenta was delivered, in addition to a normal fetus with a normal placenta. Following termination of the pregnancy, the PHM progressed into an invasive mole with lung metastasis, a rare event. Serum human chorionic gonadotrophin (hCG) concentrations decreased in the first week following delivery, but over the following 21 days hCG levels showed a continuous increase. Following 2 cycles of combinative chemotherapy consisting of fluorouracil (5-FU) and dactinomycin (KSM), hCG concentrations decreased to normal levels. The patient was then administered 1 cycle of repeated chemotherapy and hCG levels remained negative for the following 2 years.
\end{abstract}

\section{Introduction}

Gestational trophoblastic disease (GTD) is known to be associated with increased maternal age and is more commonly observed in Asia. GTD has been subdivided into partial hydatidiform mole (PHM) with a fetal pole, often with triploidy, which refers to the combination of a fetus with localized hydatidiform placenta, and complete HM (CHM) without fetal tissue, which is typically diploid but derived entirely from the paternal genome (1). CHM pregnancy is more common than PHM (1). A few cases of CHM with twin pregnancy have been reported (2-4), with certain couples choosing to continue the

Correspondence to: Dr Zhao Duan, Department of Obstetrics and Gynecology, The Second Affiliated Hospital of Xi'an Jiaotong University, Xi'an, Shaanxi 710004, P.R. China

E-mail: z.duan@yahoo.com.cn

Key words: partial hydatidiform mole, invasive mole, human chorionic gonadotrophin, lung metastasis, chemotherapy pregnancy until 28 weeks of gestation and deliver by Caesarean section, resulting in some healthy babies (2-4). In this study, we present the first reported case of a patient who was found to have PHM with a co-existing twin following in vitro fertilization, resulting in an invasive mole that progressed and metastasized to the lung.

\section{Case report}

This study was approved by the Medical Ethics Committee of Hubei University of Medicine. The patient provided informed written consent. A 34-year-old, gravida 2, para 1, patient was known to have pelvic inflammatory disease. The patient's first pregnancy was a healthy girl delivered when the patient was 20 years old. The patient then divorced at 24 and remarried at 27 . The patient's husband also had a healthy girl from his first marriage. No pregnancy occurred in the first 5 years of marriage, even though the couple were not using any contraceptive methods. The patient was then referred for assisted conception after she was diagnosed with blockage of the fallopian tubes. Following ovarian suppression with a gonadotropin-releasing hormone (GnRH) analogue, follicular growth was stimulated using purified $\mathrm{Gn}$ and ovulation was induced with human chorionic gonadotropin (hCG). Six oocytes were retrieved under transvaginal ultrasound guidance and these oocytes were then inseminated with spermatozoa at a concentration of 25,000 motile spermatozoa per oocyte. Fertilization was confirmed the following day, with 6 oocytes observed to be normally fertilized ( 2 pronuclei observed). Two 4-cell stage embryos were transferred into the uterus $48 \mathrm{~h}$ following egg collection. Progesterone intramuscular injection was used for luteal support. Six weeks following embryo transfer, 2 fetal poles, each with a positive fetal heart, were observed. At 10 weeks the 2 fetuses appeared to have a human form, as identified via transvaginal sonography (TVS).

The patient experienced vaginal bleeding at 16 weeks, and repeated TVS revealed 2 viable fetuses; however, 1 placenta was found to be cystic (Fig. 1). Serum hCG levels were found to be elevated at $800,842 \mathrm{mIU} / \mathrm{ml}$. Following careful consideration, the couple decided to terminate the pregnancy and delivery was induced with rivanol. After 37 h, 2 fetuses (230 and $249 \mathrm{~g}$ ), a normal placenta (200 g) and a partial cystic 


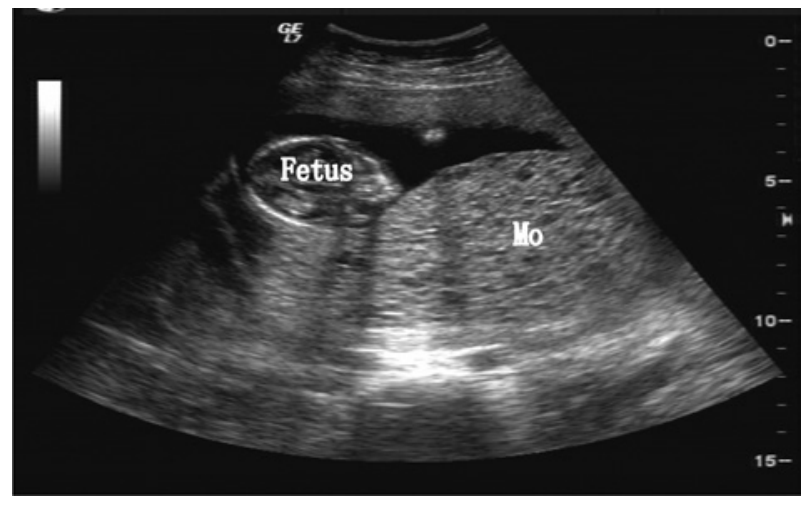

Figure 1. TVS image of the uterus showing the vesicular tissue (Mo) and the fetus. TVS, transvaginal sonography.

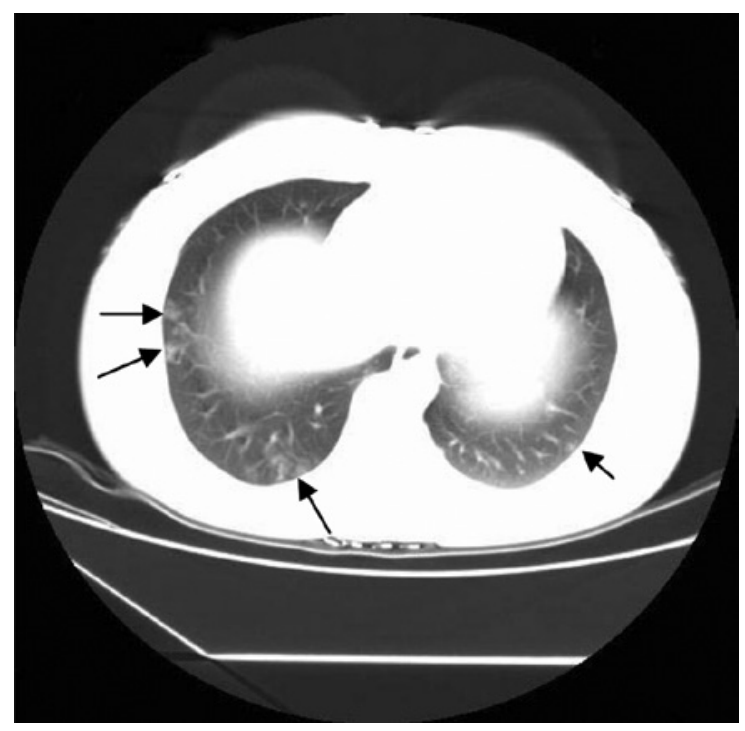

Figure 2. CT chest scan showing the lung metastasis of the trophoblast. Arrows indicate metastatic tumors. CT, computed tomography.

placenta $(290 \mathrm{~g})$ were delivered. Histological examination confirmed the clinical impression of 1 normal placenta and a second partial HM (69, XXY). Three weeks following curettage, serial serum hCG concentrations increased continually, and TVS indicated that the trophoblast had invaded the uterine myometrium. An X-ray computed tomography (CT) chest scan indicated that the trophoblast had metastasized (Fig. 2). Following 3 cycles of chemotherapy consisting of fluorouracil (5-Fu) $(26 \mathrm{mg} / \mathrm{kg} / \mathrm{day}, 8$ day/cycle) and dactinomycin (KSM) ( $6 \mathrm{ug} / \mathrm{kg} / \mathrm{day}, 8$ day/cycle), the metastases disappeared and hCG levels became negative, remaining normal for 1 year (Fig. 3).

\section{Discussion}

HM results in abnormal pregnancy with excessive proliferation of placental villi but severely stunted or absent embryonic development. HMs should be regarded as premalignant lesions since $15-20 \%$ of CHMs and $1 \%$ of PHMs undergo malignant transformation into invasive moles, choriocarcinomas or, in rare cases, placental-site trophoblastic tumors (PSTTs) (5). Metastases in gestational trophoblastic neoplasia (GTN)

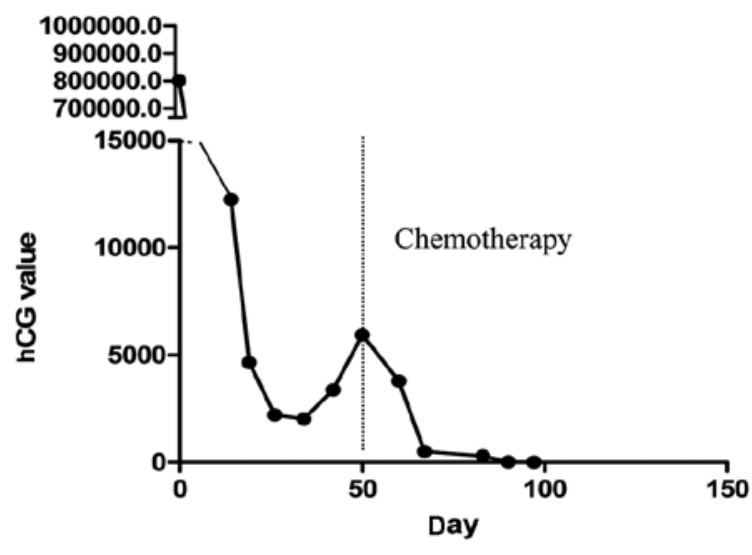

Figure 3. Serial measurements of serum human chorionic gonadotrophin (hCG) concentrations prior to delivery. The first hCG value was $800,842 \mathrm{mIU} / \mathrm{ml}$. Following chemotherapy, hCG values remained in the normal range.

develop in approximately $4 \%$ of cases following the evacuation of CHMs, while fewer than 5\% of PHMs are likely to develop postmolar GTN. Metastases rarely occur and a histopathological diagnosis of choriocarcinoma has not previously been confirmed following a partial mole (5). A total of 10-17\% of HMs result in an invasive mole, and approximately $15 \%$ of these metastasize to the lungs or vagina (6).

Although recent advances in TVS may help to diagnose a twin molar pregnancy commencing at the end of the first trimester, diagnosis of PHM based on morphological observation is sometimes difficult and inaccurate (5-6). However, in the present case, the TVS scan clearly revealed a normal placenta and sharply defined molar tissue. An invasive mole is most often clinically diagnosed rather than pathologically, based on persistent hCG elevation following molar evacuation, and is frequently treated with chemotherapy without a histopathological diagnosis (5). In China, combination chemotherapy consisting of 5-FU and KSM is used as an effective treatment for GTN (7).

Assisted reproductive technology has enhanced the fertility of older females, which may increase the proportion of GTD cases in this age group. This increase is of concern since malignant sequelae occur more frequently in older patients (8). However, it is most likely that certain clinical factors (advanced maternal/ paternal age and/or poor oocyte quality) that precipitate in vitro fertilization (IVF) treatment also predispose these patients to GTD. For instance, if 2 spermatozoa or a diploid sperm enter an oocyte, the result is triploid tissue. It has been established that some spermatozoa remain diploid, particularly as paternal age increases.

Following the diagnosis of HM and co-existing fetus, the decision to terminate or continue the pregnancy was a difficult one. However, a previous study has demonstrated that only three patients out of $14(21 \%)$ delivered a healthy child in those circumstances, and that these patients potentially face a higher risk of GTN than CHM (4). For patients with PHM with a co-existing fetus, few have full-term infants (4). It has been suggested that medical induction of labor and hysterotomy are not recommended for molar evacuation since these methods increase maternal morbidity, including blood loss, incomplete evacuation requiring dilation and curettage, and the require- 
ment for Caesarean delivery in subsequent pregnancies. These treatments also increase trophoblastic dissemination and the development of postmolar GTN requiring chemotherapy (9-10). However, since bones of the fetus have formed, a bone spur may penetrate the uterus or cervix and cause severe and/or heavy bleeding during clamping. These possibilities should also be considered. We hypothesize that rivanol induction contributes to patient progression into invasive mole and metastasis of the lung. Therefore, determination of the best method for terminating these pregnancies requires further investigation.

Twin pregnancies with a molar pregnancy and co-existing viable fetus have been described (4,11-13). The majority of pregnancies were terminated once the diagnosis was made. However, a few reports have shown that these patients completed a successful pregnancy following CHM diagnosis. It remains unclear as to whether a greater risk of postmolar disease is associated with more aggressive behavior of the molar tissue or with delayed delivery. Certain reports suggest that prolonging gestation does not appear to increase HM malignant changes (5). Since PHM results in milder clinical symptoms and a reduced incidence of malignant transformation than CHM, we suggest that it may be safe to continue these pregnancies, provided that the fetal karyotype is normal and maternal complications are controlled.

\section{References}

1. Vassilakos P and Kajii T: Letter: Hydatidiform mole: two entities. Lancet 1: 259, 1976.
2. Montes-de-Oca-Valero F, Macara L and Shaker A: Twin pregnancy with a complete hydatidiform mole and co-existing fetus following in-vitro fertilization: case report. Hum Reprod 14: 2905-2907, 1999

3. Lee SW, Kim MY, Chung JH, Yang JH, Lee YH and Chun YK: Clinical findings of multiple pregnancy with a complete hydatidiform mole and coexisting fetus. J Ultrasound Med 29: 271-280, 2010.

4. Massardier J, Golfier F, Journet D, et al: Twin pregnancy with complete hydatidiform mole and coexistent fetus: obstetrical and oncological outcomes in a series of 14 cases. Eur J Obstet Gynecol Reprod Biol 143: 84-87, 2009.

5. Seck1 MJ, Sebire NJ and Berkowitz RS: Gestational trophoblastic disease. Lancet 376: 717-729, 2010.

6. Lurain JR: Gestational trophoblastic disease I: epidemiology, pathology, clinical presentation and diagnosis of gestational trophoblastic disease, and management of hydatidiform mole. Am J Obstet Gynecol 203: 531-539, 2010.

7. Zhao Y, Zhang W and Duan W: Management of gestational trophoblastic neoplasia with 5-fluorouracil and actinomycin D in northern China. J Reprod Med 54: 88-94, 2009.

8. Tsukamoto N, Iwasaka T, Kashimura Y, Uchino H, Kashimura M and Matsuyama T: Gestational trophoblastic disease in women aged 50 or more. Gynecol Oncol 20: 53-61, 1985.

9. Soper JT: Surgical therapy for gestational trophoblastic disease. J Reprod Med 39: 168-174, 1994

10. Tidy JA, Gillespie AM, Bright N, Radstone CR, Coleman RE and Hancock BW: Gestational trophoblastic disease: a study of mode of evacuation and subsequent need for treatment with chemotherapy. Gynecol Oncol 78: 309-312, 2000.

11. Kajii T and Ohama K: Androgenetic origin of hydatidiform mole. Nature 268: 633-634, 1977.

12. Kim CH, Kim YH, Kim JW, et al: Triplet pregnancy with partial hydatidiform mole coexisting with two fetuses: a case report. J Obstet Gynaecol Res 34: 641-644, 2008.

13. Niemann I, Bolund L and Sunde L: Twin pregnancies with diploid hydatidiform mole and co-existing normal fetus may originate from one oocyte. Hum Reprod 23: 2031-2035, 2008. 